\title{
Philosophiques
}

\section{En hommage aux araignées... et aux femmes}

\section{Esther Rochon}

Volume 21, numéro 2, automne 1994

Les femmes et la société nouvelle

URI : https://id.erudit.org/iderudit/027285ar

DOI : https://doi.org/10.7202/027285ar

Aller au sommaire du numéro

Éditeur(s)

Société de philosophie du Québec

ISSN

0316-2923 (imprimé)

1492-1391 (numérique)

Découvrir la revue

Citer ce document

Rochon, E. (1994). En hommage aux araignées... et aux femmes. Philosophiques, 21(2), 441-451. https://doi.org/10.7202/027285ar

\section{Résumé de l'article}

Le féminisme n'est pas le propos central de l'écrivaine, bien quelle reconnaisse en profiter. Après avoir présenté l'un de ses personnages féminins principaux, qui apparaît dans une série de romans, elle la compare à l'une des figures principales du bouddhisme tibétain, une femme qui atteignit l'état de Bouddha, le propos s'oriente ensuite vers une perspective utopique, utilisant le royaume mythique de Shambhala ainsi qu'une utopie présentée dans une oeuvre de l'écrivaine. Sur un ton joyeux et un peu embarrassé, la conclusion exprime de l'indifférence à l'égard d'une variété de sujets.
Ce document est protégé par la loi sur le droit d'auteur. L'utilisation des services d'Érudit (y compris la reproduction) est assujettie à sa politique d'utilisation que vous pouvez consulter en ligne.

https://apropos.erudit.org/fr/usagers/politique-dutilisation/ 


\title{
IABIE RONDE DES É CRIVAINES
}

\section{En hommage aux araignées... et atx femmes}

\author{
par \\ Esther Rochon
}

\begin{abstract}
RESUME : Le féminisme n'est pas le propos central de l'écrivaine, bien qu'elle reconnaisse en profiter. Après avoir présenté l'un de ses personnages féminins principaux, qui apparaît dans une série de romans, elle la compare à l'une des figures principales $d u$ bouddhisme tibétain, une femme qui atteignit l'état de Bouddha. Le propos s'oriente ensuite vers une perspective utopique, utilisant le royaume mythique de Shambhala ainsi qu'une utopie présentée dans une auvre de l'écrivaine. Sur un ton joyeux et un peu embarrassé, la conclusion exprime de l'indifférence à l'égard d'une variété de sujets.
\end{abstract}

\begin{abstract}
Feminism is not the author's main concern or theme, though she profits from it. After introducing one of her main feminine characters, who appears in a series she wrote, she links her with a main figure of Tibetan Buddhism, who became a female Buddha. This is put in an utopian perspective, using the mythical kingdom of Shambhala as well as one of the author's books. In a tone both joyful and apologetic, the conclusion expresses indifference over a variety of topics.
\end{abstract}

Je m'étais présentée pour faire une communication suivie d'une lecture, munie d'un livre pour la lecture, d'un autre pour y puiser une citation et un encouragement, et d'une feuille sur laquelle j'avais écrit :

$$
\begin{aligned}
& \text { - Fém } \\
& \text { - E h a A - titre conf } \\
& \text { - AV } \downarrow \\
& \text { - Y T } \\
& \text { - Sh } \\
& \text { - Ed D - prés } \& \text { lecture }
\end{aligned}
$$


M. Guy Bouchard, co-organisateur de ce colloque, m’a demandé, après la présentation, de faire parvenir le texte de ma communication, pour les Actes. Eh bien, ce texte, le lecteur vient de le lire.

Cependant, afin d'éclairer ceux pour qui ceci demeurerait obscur, je vais développer davantage. Je le ferai en me rappelant ce que j'ai dit, et en ajoutant des commentaires.

J'ai commencé par des remerciements. J'arrivais de Montréal et, sur la route, l'autobus de dix heures avait perdu un pneu; notre conducteur (de l'autobus suivant) a fait monter des passagers et l'autre conducteur. Celui-ci a raconté toutes sortes d'histoires le long du chemin, c'était distrayant. Ensuite, j'ai traversé les centres d'achats de Ste-Foy, allant vers l'université Laval. Dans celui qui est le plus près du campus, il y avait une exposition de dessins d'Edgar P. Jacobs, comprenant de nombreux croquis de planches de bandes dessinées. On pouvait voir comment il avait travaillé, concevant chaque page comme une œuvre ayant son mouvement, sa tonalité, ceci indépendamment du texte ou de la couleur. Je ne m'attendais pas à me trouver nez à nez avec quelque chose d'aussi beau. Ensuite, de l'autre côté de la rue,j'ai emprunté un joli sentier dans le bois, débouchant à côté de la résidence des jeunes filles, puis j'ai fait mon chemin sur le plateau battu des vents, pour me retrouver au lieu de la rencontre. Tout ceci étant agréable et varié, des remerciements me semblaient de mise.

\section{Fém}

Sous cette abréviation de féministe se place une remarque : lors de l'annonce du colloque, vu que ma communication se plaçait avec celles de Louky Bersianik et d'Elisabeth Vonarburg, dans son annonce le journal Le Devoir avait parlé de nous comme d'écrivaines féministes. J'ai tenu à rectifier que, pour ma part, une ou un féministe est quelqu'un qui fait avancer concrètement la cause des femmes, que j'étais honorée d'un tel qualificatif, sans toutefois voir en quoi je le méritais.

\section{EH a A - titre conf}

En Hommage aux araignées, noté E H a A sur ma feuille, est le titre de mon premier roman. Le titre de ma communication (titre conf), déterminé au téléphone avec Guy Bouchard, était « En Hommage aux araignées - et aux femmes ». Lors de la communication ${ }^{\mathrm{I}}$, je me suis sentie tenue d'expliquer brièvement ce titre, comme suit. J'écris des livres dans lesquels se trouvent des personnages féminins. Parmi ces livres, En Hommage..., pilonné depuis une dizaine d'années mais réédité dans sa version jeunesse, LEtranger sous la ville. Parmi ces personnages...

I. Comme dans le titre, p. 207. 


\section{$A \vee \downarrow$}

Parmi ces personnages, donc, Anar Vranengal (notée ici A V), qui a douze ans et est apprentie sorcière dans ce texte, et qu'on retrouve dans les deux autres livres de la série, LÉpuisement du soleil et surtout L'Espace du diamant. La petite demoiselle de douze ans est en fait protagoniste et narratrice d'En Hommage..; de plus, au cours des trois livres je l'ai suivie, m'identifiant souvent à elle. (Je m'identifie presque toujours à mes personnages, hommes, femmes ou autres, comme je l'ai écrit en Ig8o dans le numéro 3 de la revue de sciencefiction québécoise Imagine...; cependant, lors de la communication j'ai omis cette précision, pour des raisons qui seront examinées plus loin). Au cours d'une quinzaine d'années de rédaction, j'ai eu le plaisir de voir Anar Vranengal s'épanouir comme sorcière en bonne et due forme, réussissant des choses qui me sembleraient à moi impossibles.

Jouvrirai ici une parenthèse. Tandis que je donnais ma communication, j'avais l'impression que la salle frissonnait chaque fois que je prononçais ce mot de sorcière, sans doute parce qu'il a été utilisé et repris dans des textes féministes. Je trouvais plutôt drôle de provoquer ainsi un effet à bon marché, dû sans doute au fait que, mon écriture n'étant connue que de peu de personnes présentes, peu savaient ce que j'avais voulu dire par là.

Jai ensuite fait un lien - dont je ne suis pas satisfaite mais enfin il fallait remplir un peu - entre l'aspect « araignées » de mon titre, le flou que leurs toiles donnent aux espaces qu'elles ornent, et le flou de ma pensée (enfin, c'est ce dont je me rappelle; c'est flou, justement). Ce lien, dans mon plan, est représenté par la flèche pointant vers le bas, en fait vers l'item suivant. J'ai sans doute dit que la sorcière Anar Vranengal ne tient pas uniquement de moi, mais de quelqu'un que j'admire beaucoup. Dans l'extrait de roman dont j'ai fait lecture après ma communication, cette filiation n'apparaît que de manière indirecte, que je préciserai plus tard ici; en ce sens, l'extrait n'était pas le mieux choisi.

\section{Y I}

Cette personne que j'admire beaucoup, c'est Yeshé Tsogyel (d'où le Y T cidessus), une dame tibétaine qui a atteint l'état de Bouddha. C'était une des épouses (dans la communication, j'ai dit l'épouse; en langage parlé j'ai tendance à simplifier) du maître bouddhiste Padmasambhava. Cette dame Sagesse Océane Royale - ce qui serait une façon de traduire son nom - atteignit l'état d'Eveil dans un style qui me semble typiquement féminin. Par exemple, jeune étudiante en mission au Népal, allant seule sur la route avec un kilo d'or, elle se vit attaquée par sept voleurs. Elle commença par s'adresser à eux en tibétain (p. 45, cf. références), leur faisant remarquer qu'ils faisaient partie de sa vision sacrée du monde et que la rencontre n'était pas fortuite. Devant la sallej'ai traduit de l'anglais, en adaptant un peu pour ne pas dérouter par la terminologie bouddhiste, des extraits du livre. Ici, je vais me passer d'adaptation : les 
personnes qui le désirent pourront consulter les commentaires qui se trouvent dans le même livre, ou ailleurs.

ô sept Yidams d'Erong

Quelle heureuse coïncidence que nous nous rencontrions aujourd'hui.

Afin que je puisse atteindre l'état de Bouddha

Et accomplir les aspirations des êtres doués de sensibilité

Puisse cette mésaventure du karma être rapidement transformée.

Découvrir, de manière fortuite, la compassion du Lama -

Quelle authentique merveille!

Des pensées heureuses surgissent de mon cœur

Puissent tous trouver la libération par la générosité.

Puis elle leur offre l'or, de bon cœur. Comme ils n'ont pas compris ce qu'elle disait, ne parlant pas tibétain, et qu'ils sont désarçonnés par son comportement, ils lui demandent d'où elle vient, qui est son père, sa mère, etc., selon la tradition de ces pays. S'exprimant sans hésiter dans leur langue, elle les renvoie à leur propre état d'esprit (encore p. 45) :

Vous, sept voleurs avec lesquels j'ai un lien karmique,

Agression et malice sont la sagesse-semblable-au-miroir elle-même -

Rayonnement et clarté n'ont d'autre source

Qu'un esprit hostile, plein de colère et d'inimitié.

Regardez dans votre colère

Et s'y trouve la force de l'être de diamant, Vajrasattva !

Détachés des apparences, vous voilà purifiès dans la vacuité.

Le pays natal de la jeune fille que je suis est Joie débordante,

Champs sereins de vacuité et de plaisir visionnaire;

Je ne tiens pas aux noms et formes conventionnelles

Et si vous attire la belle contrée de cette jeune fille que vous aimez,

Je vous y conduirai.

Elle poursuit, faisant correspondre les cinq familles-de-bouddha aux cinq questions qu'ils lui ont posées. La citation qui précède a trait à la famille Vajra, associée à l'agression; par exemple, pour Ratna, associée à l'orgueil, elle note (p. 46) :

La pureté primordiale dans la méditation ne peut être trouvée

Qu'en un esprit ambitieux qui se prend pour le meilleur de tous.

Et ainsi de suite. Les voleurs, comprenant ce qu'elle dit, lui demandent d'autres enseignements et des préceptes, qu'elle leur donne, ce qui les libère du samsara, et elle poursuit son chemin avec son kilo d'or.

Plus tard, étant parvenue à un niveau supérieur de réalisation, elle fera des choses plus difficiles. Par exemple, elle fut un jour volée et violée par sept bandits, après quoi elle leur chanta une introduction aux quatre joies (p. Iı8-iıg), leur montrant comment l'acte sexuel avec elle les avait fait passer par des états 
d'esprit associés à l'Éveil, ce qui leur fit atteindre sur-le-champ un état spirituel très avancé, celui de Mahasiddha, qui leur permit de faire le bien des autres.

J'ai poursuivi mon exposé par les remarques d'usage, à savoir :

- ne vous lancez pas dans l'imitation de ces exemples, à moins de vraiment savoir ce que vous faites;

- Yeshé Tsogyel a-t-elle vraiment existé ? Ceci lui est-il vraiment arrivé ? Impossible de remonter dans le temps pour le savoir. Ce qui importe ici, c'est que son histoire nous parvienne, et, avec elle, des manières nouvelles de répondre aux agressions qui sont le lot des femmes, manières où l'on confronte l'agresseur à son état d'esprit, non pour lui faire honte, mais pour qu'il y saisisse le germe profond et sacré d'une sagesse qui appartient à tous, et qu'il peut ensuite propager, pour le bien commun. Que l'on soit prêt ou non à suivre un tel exemple, le simple fait de savoir qu'il existe peut être utile.

Au sujet de ce genre de méthodes, je me souviens avoir utilisé le terme « judo de l'esprit ». J'avais dû l'entendre ailleurs, et ça me semblait joli à placer. Mais en fait, je ne sais pas s'il s'applique ici, ne connaissant pas vraiment son sens. Avec cette partie de ma communication, je me sentais sur la défensive, parce qu' offrir de bon cœur ses possessions à un voleur ou à un mendiant, ou encore réaliser que celui qui nous viole - ou qui fait du harcèlement sexuel est fondamentalement bon tout comme soi-même, sont des attitudes mentales contraires à bien des convictions. Comment empêcher qu'elles ne dégénèrent en bonasserie ? Par l'exercice de sa propre intelligence de la situation, telle est la réponse classique, la compassion, c'est quelque chose d'intelligent, qui se pratique d'abord en rapport avec une situation personnelle concrète. Mais, l'autre jour, plutôt que d'aborder cet aspect, j'ai préféré, effarouchée, parler de judo de l'esprit. Franchement.

\section{Sh}

Sh tient ici pour Shambhala, pays légendaire (ou non, selon les interprètes) dont les habitants sont très sages. J'ai fait le lien entre la partie précédente et celle-ci de la manière suivante :

De même que des gens, par exemple la grande dame Yeshé Tsogyel, peuvent nous servir d'exemple ou d'inspiration personnelle, de même des pays peuvent nous inspirer au point de vue social. Shambhala, c'est une société éveillée, une société juste, et il est approprié que ce nom soit prononcé dans le cadre du thème « Les Femmes et la société nouvelle », puisque le terme «société nouvelle » a une connotation utopique, tout comme une société éveillée telle que Shambhala.

Pour en savoir plus sur le sujet, le mieux est de consulter l'ouvrage qui porte ce titre (cf. bibliographie); d'autre part, on peut aussi considérer son propre cœur : quelle serait votre idée d'une société qui fonctionne bien ? Votre vision n'est probablement pas très éloignée de celle de Shambhala. En un tel pays, du moins tel qu'on se l'imagine à partir d'ici (cette précision, quej'ai faite lors de ma communication, vaudrait d'être développée, mais je me sens un peu 
paresseuse), hommes et femmes seraient égaux de fait, tâches et richesses seraient réparties avec justice, tous auraient la possibilité de s'épanouir selon leurs talents et leurs goûts, etc. Réaliser cela concrètement, bien sûr, c'est une autre paire de manches. Comment savoir si une société juste a déjà existé ? Faute d'un exemple historique qui rallierait les opinions, il n'est pas certain qu'une telle société soit même possible. Il n'en demeure pas moins que l'aspiration demeure, et apparaît régulièrement dans les mythes, les rêves, les idéaux de différentes cultures. Non seulement cela, mais elle constitue une force d'amélioration, qui a en elle-même sa valeur.

De plus, étant moi-même impliquée dans un projet de société shambhalienne, j'ai pu, au cours des ans, faire l'expérience de certaines structures, de certaines méthodes; les personnes intéressées trouveront une adresse pertinente à la fin de ce texte.

\section{E dD - prés \& lecture}

Comme je l'ai écrit en détail dans Imagine... 58, mon roman L'Espace $d u$ diamant (noté E d D; prés \& lecture tient pour présentation et lecture) présente une société shambhalienne, le Catadial. Je me suis basée sur mon expérience, mes connaissances et mes rêveries. Pour mes collègues du milieu shambhalien, le texte contient quelques allusions pas piquées des vers, mais cet aspect fait partie de la petite histoire.

J'ai choisi d'en lire un extrait qui a suscité peu de commentaires de lecteurs, à savoir l'affrontement entre Jouskilliant Green et Anar Vranengal, p. 264 à 270. Dans ma communication, j'ai situé l'extrait, ce que j'omettrai ici, le lecteur pouvant toujours se référer au texte. D'autre part, j'ai présenté le contexte en le faussant délibérément.

Je m'adressais à des gens qui ignoraient ce quej'avais écrit, ou qui ne s'en souvenaient plus dans les détails. J'ai misé là-dessus pour biaiser la lecture, m'attendant, ce qui fut le cas, à ce que personne ne le relève. Pour cette raison, je souhaitais m'abstenir de rédiger le texte de ma communication : je me permets de prendre toutes sortes de libertés en expression orale mais, quand il s'agit d'écrit, j'aime mieux être conséquente avec mes écrits précédents. Ayant accepté de rendre compte ici de ce que j'ai dit, il m’est impossible de nier mon entourloupette. Je vais donc décrire en quoi elle consista, expliquer ma motivation, patiner un peu pour me justifier, et conclure par des remarques sans lien direct avec le sujet.

En quoi j'ai faussé le contexte : j'ai présenté la société juste du Catadial comme décrite par une femme. Il est vrai que l'extrait que j'allais lire était un dialogue entre Anar Vranengal, femme qui s'apprête à se rendre au Catadial et attend beaucoup de ce pays, et Jouskilliant Green, homme qui nie l'authenticité du Catadial en tant que société juste. Cependant, ailleurs dans le roman, le Catadial est découvert par un homme, la vision fondatrice de ce pays est définie comme venant d'hommes et de femmes (Othoum, Suzanne, etc.). Mieux encore, L'Espace du diamant comporte davantage de personnages 
masculins que de féminins et Anar Vranengal, sans y être un personnage vraiment secondaire, est loin de dominer l'action. Son rôle dans ce roman est bien moins important que celui qu'elle tient dans En Hommage aux araignées. Dans ma communication, $j$ 'ai en somme laissé entendre qu'il était naturel que l'impulsion utopique vienne d'une femme, fait avec lequel je ne suis pas d'accord, comme le roman lui-même en témoigne.

Quelle était ma motivation? Tout simplement, je me trouvais devant des gens qui s'attendaient à entendre parler en bien des femmes, et je suivais le courant. Ma tendance, en public, est de prendre la voie de la facilité. Je l'ai suivie ici; je n'avais pas envie de jouer les trouble-fête. Il y avaıt une atmosphère de célébration de la femme dans le colloque, qui avait quelque chose de convivial, de chaleureux; de ce point de vue, il me semble encore valide que j'aie préféré m'y joindre. D'autre part,j'avais l'impression que décrire franchement ma position serait décevoir, et nuire à une pensée dont je tire personnellement des avantages. Au fait, quelle est ma position ? Eh bien, le féminisme, comme l'indépendantisme, sont des sujets qui m'indiffèrent. Je n'ai pas envie de m'y impliquer.

Comment je me justifie ? Voici. L'humanité, ce sont des gens, et il y en a de toutes sortes; qu'on les classe par sexe, couleur, langue, etc., chacun est une personne, chaque situation est vécue personnellement. Les êtres m'intéressent plus que le fait qu'ils soient hommes ou femmes, qu'ils parlent français ou anglais, qu'ils soient bourgeois ou prolétaires, qu'ils viennent d'un pays riche ou d'un pays pauvre. J'ai beau m'exprimer en français, être une femme, vivre au Québec, ma place n'est pas nécessairement à défendre une langue, un sexe ou un pays. C'est peut-être un manque de maturité ou de sensibilisation de ma part, en tous cas j'en suis là.

Voilà le genre d'affirmation que je n'avais pas envie de faire en public. J'étais prête à empocher le chèque, à mettre mes beaux vêtements et à parler une demi-heure. De là à ce que je dise ce que je pense à des étrangers, des étrangères, tout de même. Par écrit, c'est connu, je suis moins timide.

Quant aux remarques sans lien direct avec le sujet, elles sont variées, et les voici.

Ce qui précède me fait penser que je mords la main qui me nourrit, et que je manque de gratitude envers les gens qui font que je parle français, les femmes qui m'ont permis d'exercer certains droits et privilèges, etc. Mais je la mords sans cesse, cette main, en toutes sortes de circonstances : je gaspille des ressources, j'occupe un territoire qui peut-être ne m'appartient pas (etc.), je gaspille ma sagesse, et personne ne me le reproche, tout cela étant si généralisé. Mordre la main qui nourrit est une constante de l'existence. Ce qui n'exclut pas qu'on puisse faire quelque chose de sa vie, malgré tout. En quels termes ? C'est un choix personnel.

Pour changer de sujet, pourquoi ai-je choisi de lire cet extrait de roman? Après tout, on n'y discute pas du Catadial; cette société juste, on ne la décrit 
pas, on ne parle pas non plus du statut de la femme, alors pour le thème « Femme et société nouvelle »...

Les personnages de cet extrait défendent des points de vue qui, regardonsy bien, relèvent des mouvements d'idées des années soixante et soixante-dix en Amérique du Nord, surtout du côté de la science-fiction. Le lecteur est sans doute aussi familier avec ces mouvements d'idées qu'avec Yeshé Tsogyel. Pour en savoir plus, il pourra consulter de récents numéros de la revue québécoise Solaris, ou encore se contenter de ce qui suit.

Dans les années cinquante et soixante, on peut dire en gros que la sciencefiction américaine était dominée par la vision de John W. Campbell, éditeur de la revue Analog, qui existe encore. La lecture de cette revue a été pour moi formatrice. On pouvait par exemple y lire les récits de Harry Harrison, réunis plus tard sous le titre Deathworld, où des scientifiques intelligents et sympa se heurtaient à des forces obscurantistes, sur fond de décors frappants. On pouvait aussi y lire Dune, de Frank Herbert, en feuilleton. Commeje ne possède pas de copie de ces ouvrages, ils ne figurent pas dans la bibliographie ci-après, mais ce sont des classiques. Dans Dune, la magie même devenait scientifique, explicable, et le décor était plus splendide que jamais. Dans un autre ordre d'idées, on pourrait dire que la science-fiction des bandes dessinées d'Edgar P. Jacobs, que je mentionnais au début, n'est pas éloignée de ce courant où le rationnel est donné comme un idéal, sans que soit exclue une imagerie fleurie. Bien sûr, Jacobs est un Européen, œuvrant sans doute sans connaître les Américains, et réciproquement, mais les flamboiements imaginaires soigneusement contrôlés par une raison qui en a la suprématie sont présents dans les deux sortes d'œuvres.

Quand j'ai commencé à écrire mon premier roman, En Hommage aux araignées, j'ai voulu présenter Jouskilliant Green comme un intellectuel campbellien, qui vient « évangéliser » les bons Asvens réfugiés sur leur Archipel minable. L'aspect décor pittoresque venait, entre autres, des caves de la Citadelle, sa résidence. J'ai constaté que la tâche m'était impossible. Je profite de la science-fiction campbéllienne comme je profite du féminisme ou de la langue française mais, là aussi,je ne suis pas sur les barricades. Ce pauvre Green devenait pour moi une figure fascinante, à la fois ridicule et puissante, un égaré rationaliste, et qui ne perd pas la raison, au sein d'un monde qui peut de temps en temps avoir honte d'être magique, mais qui ne s'en repent pas vraiment. C'est là que se situait, et que se situe encore, ma sensibilité, que je le veuille ou non. J'ai repris ce personnage dans L'Espace du Diamant, pour la scène quej'ai lue.

Dans les années soixante-dix, un nouveau courant est devenu dominant dans la science-fiction américaine et anglaise, ce qu'on a appelé le « New Wave », et qui regroupait plusieurs tendances. Dans le mouvement des idées, la science pure et dure avait montré ses limites. Place à la poésie, place au mythe! A ce moment, j'ai commencé d'écrire en suivant ce courant plutôt qu'en étant fondamentalement nourrie par lui : mes années de formation s'achevaient. 
Fait digne de mention dans notre contexte, beaucoup plus de femmes se mirent alors à écrire de la science-fiction.

Le personnage d'Anar Vranengal, à son tour, est à mon sens typique de ce second mouvement : la jeune sorcière se cherche - l'aspect psychologique étant souvent plus développé dans le «New Wave » que dans la science-fiction campbellienne. Qu'elle tienne un rôle principal dans l'un des trois livres, qui est écrit par une femme, est également représentatif de la présence accrue des femmes, écrivaines, lectrices ou personnages, dans ce mouvement. Anar Vranengal s'oriente finalement vers son état de sorcière, l'irrationnel tenant sa place dans cette nouvelle science-fiction, sous forme par exemple d'aspect anthropologique ou social. Le genre de la fantasy prit d'ailleurs un nouvel essor à cette époque, et, si mes souvenirs sont exacts, ce sont des femmes qui, en majorité, se sont mises alors à le pratiquer. (Certains disent que j'écris de la science-fiction, d'autres de la fantasy, d'autres de la littérature générale. Là aussi, le sujet m'indiffère. Je me définis comme écrivain de science-fiction parce que j'y trouve mes racines littéraires, et aussi par solidarité avec le milieu). Anar Vranengal, ce qui est également typique, n'en est pas moins rompue aux raisonnements logiques. En quoi est-ce typique? Eh bien, on parle ici de genres littéraires (science-fiction et fantasy) bien ancrés dans la société occidentale, pratiqués et lus par des gens qui s'y sentent à peu près à l'aise; donc, dans les œuvres, des marginaux peuvent figurer parce qu'ils ont une belle gueule, mais l'intelligence analytique, qui est un de nos héritages importants, ne cesse pas de fonctionner.

L'extrait de roman que j'ai lu montre l'affrontement de ces deux courants, science-fiction campbellienne et «New Wave ». Pour Green, de l'ancienne école, il y a opposition entre rationnel et magie; pour Anar Vranengal, la pensée magique qu'elle pratique contient la pensée rationnelle, l'esprit pleinement épanoui se réjouit de la richesse des raisonnements logiques.

Tout à coup, ici, on retombe en pleine pensée féminine. En effet, dans le texte lu, la position d' Anar Vranengal est axée sur deux aspects :

- le rationnel est inclus dans le magique au sens où elle le définit;

- l'affrontement avec l'autre est inclus dans l'amour qu'elle lui porte.

C'est une position imprenable; on comprend qu'elle triomphe même si elle a besoin de se ressaisir en cours de route. Et c'est une position à mon sens très féminine, pas si éloignée de celle de Yeshé Tsogyel face à ses assaillants.

J'avouerai autre chose. Je me sers de la science-fiction mais, pour ce genre littéraire, comme pour le féminisme, la langue française, le Québec, la vision campbellienne, alouette, je ne suis pas sur les barricades non plus. Je m'en sers à plus d'un titre, mais ce que j'exprime dans mes livres n'est pas l'un des messages dominants de la science-fiction, fût-elle campbéllienne, « New Wave », québécoise ou autre. D'accord, nous sommes tous des individus très individuels pour ce qui est de la pratique de l'écriture, et en ce sens ce que je viens d'affirmer ne veut pas dire grand-chose. Je préciserai donc. La science-fiction, 
genre populaire contemporain, suppose souvent un certain parti pris individualiste ou amoureux du genre « nous contre tous », une certaine dénonciation de l'autorité et du système, qui ne m'intéressent pas plus que les « alouettes » précédents.

En plus, je suis dans une position à peu près imprenable, non pour des raisons de logique comme Anar Vranengal, mais à cause de l'apathie extérieure. J'écris ce que je veux, je peux faire de la propagande pour le bouddhisme ou pour Shambhala, dissimulée que je suis derrière mon immunité d'écrivaine qui se débrouille avec les mots. Mon activité demeure anodine : le message, la plupart du temps, ne se rendra pas. La plupart des gens qui transmettent des idées ou qui écrivent se trouvent dans une situation analogue, à juste titre ou non. Ça fait partie de la forme du monde. Mais aussi mon attitude vis-à-vis de la survie du français, des droits de la femme, de la contestation du pouvoir établi, etc., est semblable à celle d' Anar Vranengal devant Green : il y a de l'amour, il y a de la gratitude, mais il n'y a pas de communion. On ne se refait pas.

Parmi les points non directement liés au sujet, voici le dernier. Dans ces romans sur Vrénalik, Jouskilliant Green, en plus d'être rationaliste, est traducteur. Par ce sceptique passent les légendes de l'Archipel, pour être rendues en langue du Sud, sous forme de littérature de gare. Je dois dire que je suis fière de cette image. Non seulement cela, mais une des raisons pour lesquelles j'ai choisi de lire ce que j'ai lu, c'est qu'on y trouvait une des phrases dont je suis la plus fière (p. 269, prononcée par Anar Vranengal) :

Les sorciers, comme les traducteurs, sont éternellement discrets, cachés dans les feuillages des rives tandis que des peuples réalisent leurs rêves.

Pourquoi cette phrase me plaît-elle tant? Parce que, dans son ambiguitéles rêves de qui ? Des sorciers, des traducteurs ou des peuples? - elle montre ma situation. Qu'est-ce que je dis au juste? Est-ce que je parle au nom de la langue française, des femmes, du Québec, de la science-fiction, de moi-même, du bouddhisme, de Shambhala, ou d'autre chose? J'ai l'impression d'une réalité chatoyante, où parfois je parle pour l'une, ou pour l'autre, me cachant derrière de beaux vêtements ou de belles phrases, ne cherchant même plus à me saisir. Entre-temps passe sur le fleuve un étrange bateau à l'étrave noircie, cependant rouge comme la passion.

\section{Références*}

Sky Dancer - The Secret Life and Songs of the Lady Yeshe Tsogyel, Keith Dowman, London, Arkana (Penguin Group), 1984.

Shambhala, Chögyam Trungpa Rinpoché (traduit de l'anglais), Paris, Seuil, Points/ Sagesses.

* Tous les titres, sauf les deux premiers, renvoient aux œuvres d'Esther Rochon. Par ailleurs, les personnes intéressées à Shambhala peuvent s'adresser au $5505 \mathrm{St}^{-}$ Laurent, Montréal H2T IS6, tél (514) 2799115. 
En Hommage aux araignées, Montréal, Éditions de l' Actuelle, I974.

L'Épuisement $d u$ soleil, Longueuil, Éditions du Préambule, (coll. Chroniques du futur, no 8), ig85.

LEtranger sous la ville, Montréal, Éditions Paulines, ig86. Version pour la jeunesse, remaniée par l'auteur, de En Hommage aux araignées.

L'Espace du diamant, Montréal, Montréal, Éditions de la pleine lune, I990.

« Notes sur L'Épuisement du soleil », Imagine... 3, I980, p. $23-28$.

«Oser actualiser l'utopie », Canadian Woman Studies/Les Cahiers de la femme, vol. 6 , $\mathrm{n}^{\circ} 2,1985$, p. 66-68.

« Notes sur L'Epuisement du soleil, zème partie : paysages et lieux d'écriture », Imagine... 3I, 1985, p. 94-IOI.

«Les Voies d'une utopie », sur l'Espace du Diamant, d'après des questions d'Hélène Colas, Imagine... 58, I99I, p. 66 à 8I. 